\title{
Plantas Daninhas em Ilhas de Vegetação em Processo de REGENERAÇÃo NATURAL ${ }^{1}$
}

\author{
Weeds on Vegetation Islands under Natural Regeneration
}

SOUZA, P.F. ${ }^{2}$, SIQUEIRA, T.C. ${ }^{3}$ e MARTINS, R.L. ${ }^{4}$

\begin{abstract}
RESUMO - Nste trabalho é apresentada a composição florística e estrutural de ilhas de vegetação de um inselberg do Vale do Moxuara, localizado no município de Cariacica-Espírito Santo, onde avaliou-se a riqueza das ilhas de vegetação está relacionada com seu tamanho e com a profundidade do solo. Foram avaliadas 45 ilhas de vegetação para estimativa dos parâmetros fitossociológicos necessários às análises. Foram encontradas 53 espécies de plantas vasculares, pertencentes a 24 famílias e 48 gêneros. As famílias com maior representatividade foram Poaceae, com sete espécies, seguida de Asteraceae, Malvaceae e Fabaceae, com seis espécies cada. Plantas daninhas representaram 39,6\% da flora inventariada e $25,5 \%$ da cobertura vegetal das ilhas. As espécies com maior frequência e cobertura relativa foram Selaginella convoluta e Anemia tomentosa. A regressão múltipla não se mostrou significativa, o que foi discutido considerando o papel inibidor de espécies daninhas.
\end{abstract}

Palavras-chave: afloramentos rochosos, inselbergs, ilhas xéricas.

\begin{abstract}
This study presents the floristic and structural composition of vegetation islands of an inselberg in Vale do Moxuara, in Cariacica-ES, to evaluate whether the islands' richness is related to their size and soil depth. A total of 45 vegetation islands were evaluated by estimating the phytosociological parameters needed for the analysis. Fifty-three vascular plant species of twenty-four families and forty-eight genders were found. The most outstanding families were Poaceae, with seven species, followed by Malvaceae and Fabaceae, with six species each. Weeds represented $39.6 \%$ of all flora listed and $25.5 \%$ of the islands' vegetation cover. Selaginella convoluta and Anemia tomentosa had the most frequency and relative cover. Multiple regression was not significant, considering the inhibiting role played by the weed species.
\end{abstract}

Keywords: rock outcrops, inselbergs, xerophytic islands

\section{INTRODUÇÃO}

Afloramentos rochosos que se apresentam como rochas isoladas que emergem abruptamente acima das planícies que os cercam são classificados como inselbergs (Porembski, 2007). Os inselbergs geralmente apresentam vegetação restrita a áreas planas, como platôs e/ou pontos e fendas, onde é possivel a germinação de sementes e o acúmulo de substratos, de forma que a vegetação se dispõe em ilhas. Uma ilha de vegetação pode ser definida como um agrupamento vegetal com uma ou mais espécies de plantas vasculares fixadas em solos rasos ou diretamente na rocha, estando esse agrupamento delimitado por uma superfície rochosa (Conceição et al., 2007).

Recebido para publicação em 6.10.2010 e aprovado em 2.3.2011.

2 Bióloga, Mestranda do Programa de Pós-Graduação Botânica - CPBO, Instituto Nacional de Pesquisas da Amazônia - INPA, Av. André Araújo, 2936, 69060-001, Manaus-AM, Brasil, bolsista CNPq; ${ }^{3}$ Bióloga, ${ }^{4}$ Professor Adjunto da Universidade Federal do Rio de Janeiro - UFRJ, Núcleo de Pesquisas em Ecologia e Desenvolvimento Sócio-Ambiental de Macaé - NUPEM, Rua Rotary Club, s/no. São José do Barreto, Caixa Postal 119331, 27910-970 Macaé-RJ, Brasil, <rodr.lemes@gmail.com>. 
Ilhas de vegetação em inselbergs tendem a apresentar uma riqueza vegetacional determinada pela dificuldade de colonização, definida pelo grau de isolamento e pelas suas características ecológicas, citando-se como exemplo o vento, a profundidade do solo, a altitude, etc. Por conta dessas características, as ilhas de vegetação fornecem um modelo experimental útil à compreensão do processo de sucessão ecológica e dos fatores que interferem nesse processo e das teorias que explicam padrões de distribuição da diversidade (Porembski, 2007).

Trabalhos em inselbergs brasileiros, geralmente, se propõem a estudar a diversidade das ilhas em áreas conservadas (Conceição \& Giulietti, 2002; Ribeiro \& Medina, 2002), sendo poucos os estudos que procuram compreender o processo de sucessão (Oliveira et al., 2004; Conceição et al., 2007; Conceição \& Pirani, 2007) e a sensibilidade delas à invasão por espécies exóticas associadas à atividade humana, principalmente plantas daninhas (Porembski, 2007). A identificação desse processo de invasão e das espécies envolvidas seria o primeiro passo para compreender quais são as circunstâncias que favorecem o desenvolvimento das daninhas e garantir o controle destas, visando ao restabelecimento de espécies nativas e à conservação de ecossistemas específicos.

Nesse sentido, objetivou-se no presente trabalho realizar um levantamento florístico em ilhas de vegetação do inselberg do Vale do Moxuara, localizado na região rural do município de Cariacica-ES, e, com base na composição e no padrão de riqueza das ilhas de vegetação, avaliar a regeneração natural da área e a influência de plantas daninhas.

\section{MATERIAL E MÉTODOS}

O estudo foi realizado em ilhas de vegetação, em um paredão rochoso, na face norte de um inselberg conhecido como Moxuara, localizado no municipio de Cariacica-ES. Esse inselberg é um afloramento granítico-gnáissico de origem pré-cambriana, típico da Serra da Mantiqueira, com aproximadamente $650 \mathrm{~m}$ de altitude, que pode ser avistado de diferentes pontos da região da Grande Vitória (RADAMBRASIL, 1983). Dados para a região do município de Cariacica revelam uma temperatura média anual variando entre 22 e $24{ }^{\circ} \mathrm{C}$ (Pezzopane et al., 2004).

Foram estudadas 45 ilhas de vegetação de um paredão rochoso de $6.900 \mathrm{~m}^{2}$, a aproximadamente $60 \mathrm{~m}$ de altitude, nas coordenadas de $20^{\circ} 17^{\prime} 14^{\prime \prime}$ sul e $40^{\circ} 26^{\prime} 23^{\prime \prime}$ oeste. Foram consideradas apenas as ilhas de vegetação sujeitas a uma inclinação entre 21 e 41 graus, com diâmetro maior ou igual a $0,5 \mathrm{~m}$. As ilhas amostradas pertenciam a uma propriedade particular destinada à caprinocultura e ecoturismo, mas que desde 2001 está isolada para recuperação por regeneração natural.

As coletas para o estudo florístico foram realizadas no período de março a agosto de 2008 e de setembro a novembro de 2009, durante visitas às ilhas estudadas. Foram coletadas todas as plantas que floresceram no período de estudo e plantas com características vegetativas distintivas que não floresceram no periodo. Apenas as plantas floridas foram herborizadas e identificadas com base na literatura e na coleção botânica do Herbário Central da Universidade Federal do Espírito Santo (VIES). O sistema adotado para descrição das angiospermas foi o proposto em APG II (Angiosperm Phylogeny Group, 2003); já para descrição de pteridófitas seguiu-se a classificação de Smith et al. (2006). Foram consideradas como plantas daninhas as espécies relacionadas por Lorenzi (2000) e Kissman \& Groth (1997), que as definem como espécies invasoras que possuem reconhecido papel de interferência sobre outras espécies, em especial sobre culturas agrícolas, por meio da competição e alelopatia.

Na análise das diferenças na estrutura da vegetação das ilhas foi utilizado o método de intercepto de linha, com valores lineares (Brower \& Zar, 1984). No centro de cada unidade insular foram alocados dois interceptos de $50 \mathrm{~cm}$ cada um, sendo um fixado no diâmetro maior da ilha e o outro perpendicularmente ao primeiro. Os parâmetros fitossociológicos utilizados para análise da composição florística das ilhas foram calculados conforme Brower $\&$ Zar (1984), sendo estes: cobertura absoluta de cada espécie (CA) - dada pela soma do comprimento interceptado por cada espécie nas ilhas medidas; cobertura relativa de cada espécie (CR) - resultado da divisão da CA pela soma das $\mathrm{CAs}$ de todas as espécies; frequência 
absoluta de cada espécie (FA) - dada pelo número de ilhas em que uma determinada espécie ocorreu; e frequência relativa de cada espécie (FR) - resultado da divisão da FR pela soma das FRs de todas as espécies.

A riqueza inventariada nos interceptos de linha de tamanho fixo foi correlacionada com a área de cada ilha e sua profundidade máxima, por meio de regressão múltipla. A profundidade do solo de cada ilha foi definida como a maior medida obtida dentre 10 diferentes mensurações feitas aleatoriamente na ilha, por meio de uma régua de metal graduada em milímetros. A área aproximada da ilha foi obtida pelo cálculo da área de uma elipse, a partir do diâmetro maior e menor da ilha.

\section{RESULTADOS E DISCUSSÃO}

Ao todo, foram encontradas 53 espécies de plantas vasculares, pertencentes a 24 familias e 48 gêneros (Tabela 1). A familia com maior número de espécies foi Poaceae, com sete, seguida de Asteraceae, Malvaceae e Fabaceae, com seis, e Euphorbiaceae, com quatro.

Tabela 1 - Relação das espécies identificadas no paredão rochoso de um inselberg localizado na Fazenda Estância Vale do Moxuara, Cariacica-ES, ano de 2009

\begin{tabular}{|c|c|}
\hline Família/espécie & Família/espécie \\
\hline $\begin{array}{l}\text { Agavaceae } \\
\text { Agave americana } \mathrm{L} .\end{array}$ & $\begin{array}{l}\text { Malvaceae } \\
\text { Malvastrum sp. }\end{array}$ \\
\hline $\begin{array}{l}\text { Asteraceae } \\
\text { * Achyrocline satureoides (Lam.) DC. } \\
\text { * Ageratum conyzoides L. } \\
\text { * Baccharis trinervis (Lam.) Pers. } \\
\text { * Emilia sonchifolia (L.) DC. } \\
\text { Lourteigia ballotifolia (Kunth) R. King \& H. Robinson } \\
\text { * Vernonia scorpioides (Lam.) Pers. }\end{array}$ & $\begin{array}{l}\text { Melochia } \text { sp. } \\
\text { * Sida cordifolia } \mathrm{L} . \\
\text { * Sida rhombifolia } \mathrm{L} . \\
\text { * Waltheria americana (L.) } \\
\text { Wissadula } \text { sp. } \\
\text { Orchidaceae } \\
\text { Prescottia nivalis } \text { Barb. Rodr. }\end{array}$ \\
\hline $\begin{array}{l}\text { Bromeliaceae } \\
\text { Tillandsia stricta (Sol. Ex Sims) }\end{array}$ & $\begin{array}{l}\text { Sacoila lanceolata (Aubl.) Garay } \\
\text { Passifloraceae }\end{array}$ \\
\hline $\begin{array}{l}\text { Cactaceae } \\
\text { Coleocephalocereus fluminensis (Miq.) Backeb. }\end{array}$ & $\begin{array}{l}\text { Passiflora foetida } \mathrm{L} . \\
\text { Phyllanthaceae }\end{array}$ \\
\hline $\begin{array}{l}\text { Cannaceae } \\
\text { Canna edulis Kerr-Gawler }\end{array}$ & $\begin{array}{l}\text { Phyllanthus tenellus Roxb. } \\
\text { Poaceae }\end{array}$ \\
\hline $\begin{array}{l}\text { Commelinaceae } \\
\text { Commelina erecta } \mathrm{L} .\end{array}$ & $\begin{array}{l}\text { Andropogon sp. } \\
\text { Digitaria } \text { sp. }\end{array}$ \\
\hline $\begin{array}{l}\text { Convolvulaceae } \\
\text { Evolvulus exilis Meisn. } \\
\text { Jacquemontia sphaerostigma (Cav.) Rusby }\end{array}$ & $\begin{array}{l}\text { * Melinis minutiflora } \text { P. Beauv } \\
\text { * Panicum maximum Jacq. } \\
\text { * Paspalum plicatulum Michx. }\end{array}$ \\
\hline $\begin{array}{l}\text { Cyperaceae } \\
* \text { Bulbostylis capillaris (L.) C. B. Clarke } \\
\text { Cyperus hermafroditus (jacq.) standl }\end{array}$ & $\begin{array}{l}\text { * Pennisetum clandestinum Hochst. Ex Chiov. } \\
\text { * Rhynchelytrum repens (Willd.) C.E.Hubb. } \\
\text { Portulacaceae }\end{array}$ \\
\hline $\begin{array}{l}\text { Euphorbiaceae } \\
* \text { Cnidoscolus urens (L.) Arthur } \\
\text { * Croton glandulosus L. } \\
\text { * Croton lundianus M. Arg. } \\
\text { Chamaesyce sp. }\end{array}$ & $\begin{array}{l}\text { Portulaca perennis R. E. Fries } \\
\text { Pteridaceae } \\
\text { Doryopteris lorentzii (Hieron.) Diels } \\
\text { Rubiaceae } \\
\text { Staelia thymoides Cham. \& Schlecht. }\end{array}$ \\
\hline $\begin{array}{l}\text { Fabaceae } \\
\text { * Aeschynomene americana } \mathrm{L} . \\
\text { Centrosema brasilianum (L.) Benth } \\
\text { Centrosema virginianum (L.) Benth } \\
\text { * Desmodium barbatum (L.) Benth. Et Oersted } \\
\text { * Stylosanthes viscosa (L.) Sw. } \\
\text { Zornia reticulata } \text { Sw. }\end{array}$ & $\begin{array}{l}\text { Anemiaceae } \\
\text { Anemia ciliata Presl. } \\
\text { Anemia tomentosa (Sav.) Sw. } \\
\text { Selaginellaceae } \\
\text { Selaginella convoluta (Arn.) Spring } \\
\text { Urticaceae } \\
\text { Cecropia hololeuca Miq. }\end{array}$ \\
\hline $\begin{array}{l}\text { Gesneriaceae } \\
\text { Sinningia brasiliensis (Regel \& Schmidt) Wiehler \& Chautems }\end{array}$ & $\begin{array}{l}\text { Verbenaceae } \\
\text { Lantana } \mathrm{sp} .\end{array}$ \\
\hline $\begin{array}{l}\text { Hypoxidaceae } \\
* \text { Hypoxis decumbens } \mathrm{L} .\end{array}$ & Lippia dulcis Trevir. \\
\hline
\end{tabular}

* Espécies daninhas invasoras, segundo Lorenzi (2000) e Kissman \& Groth (1997). 
Em trabalhos realizados nos Estados de Sergipe e Bahia, as famílias com maior número de espécies foram Poaceae e Asteraceae (Conceição \& Giulietti, 2002; Ribeiro et al., 2007). Em afloramentos do Estado de São Paulo, Fabaceae, Asteraceae e Poaceae foram as famílias com maior representatividade (Oliveira \& Godoy, 2007). No entanto, os estudos supracitados foram realizados em ilhas de vegetação com espécies nativas, ao contrário do inselberg do Moxuara, em que a maioria das espécies das famílias Poaceae e Asteracea registradas são plantas daninhas invasoras (Lorenzi, 2000). Essa semelhança na ocorrência de famílias está relacionada à facilidade de alguns grupos vegetais na colonização desses ambientes rigorosos, sujeitos a elevado grau de insolação, também conhecidos como "ilhas xéricas" (Gröger \& Barthlott, 1996).

O número de plantas daninhas registradas nas ilhas de vegetação representou $39,6 \%$ do total de espécies inventariadas (Tabela 1). As espécies relacionadas como daninhas são consideradas eficientes competidoras e colonizadoras de áreas degradadas, desenvolvendose mesmo em condições xéricas e em solos oligotróficos, o que está relacionado à eficiência delas na utilização de água e nutrientes (Modesto-Júnior \& Mascarenhas, 2001; Ferreira et al., 2002; Yamashita et al., 2009).

$\mathrm{Na}$ avaliação da estrutura da vegetação do conjunto de ilhas analisadas, por meio do intercepto de linha, foram registradas 21 espécies (Tabela 2), aproximadamente $40 \%$ do total de plantas inventariadas. As espécies plantas daninhas representaram $25,5 \%$ da cobertura vegetal das ilhas de vegetação e, aproximadamente, $50 \%$ das espécies inventariadas pelo intercepto de linha. Ageratum conyzoides foi a espécie daninha com maior cobertura relativa: 4,84\%. Segundo Macedo et al. (2006), essa espécie apresenta alta densidade relativa na maioria das áreas alteradas por culturas agrícolas, sendo essa dominância modificada pelo uso de técnicas de manejo específicas.

Selaginella convoluta e Anemia tomentosa destacaram-se pelos maiores valores de cobertura relativa: $54,8 \%$ e $8,24 \%$, respectivamente. Essas duas espécies foram encontradas sozinhas, formando ilhas monodominantes, em uma estrutura em forma de "tapete". Essas estruturas, possivelmente, estariam servindo como substrato para outras espécies, que não conseguem se afixar diretamente sobre a rocha exposta, semelhantemente aos tapetes de monocotiledôneas referidos por Caiafa \& Silva (2007) em inselbergs do Sudeste do Brasil.

Segundo Porembski (2007), ao contrário de outras Selaginellaceae, S. convoluta é, geralmente, rupícola, crescendo sobre uma fina camada de solo nas encostas de morros e paredões rochosos, onde forma "tapetes". Para esse autor, as ilhas iniciadas a partir de Selaginella têm aumento gradual na quantidade de matéria orgânica e sedimento, favorecendo a inclusão de arbustos e arvoretas, onde o solo já está estabelecido. Esse padrão de aumento da riqueza a partir de condições criadas por uma ou poucas espécies também foi definido para Velloziaceae por Scarano (2002) e Conceição et al. (2007).

A relação da riqueza obtida nos interceptos de tamanho fixo com o tamanho das ilhas e com a profundidade não foi significativa

Tabela 2 - Parâmetros fitossociológicos das espécies amostradas nas ilhas de vegetação do afloramento rochoso da Fazenda Estância Vale do Moxuara, Cariacica-ES, ano de 2009, e as respectivas frequências absolutas (FA), frequências relativas (FR), coberturas absolutas (CA) e coberturas relativas (CR)

\begin{tabular}{|c|c|c|c|c|}
\hline Espécie & FA & FR $(\%)$ & $\mathrm{CA}(\mathrm{m})$ & CR (\%) \\
\hline Selaginella convoluta & 0,87 & 30,95 & 0,654 & 54,80 \\
\hline Anemia tomentosa & 0,44 & 15,87 & 0,098 & 8,24 \\
\hline Bulbostylis capillaris* & 0,22 & 7,94 & 0,053 & 4,46 \\
\hline Sinningia brasiliensis & 0,29 & 10,32 & 0,020 & 1,62 \\
\hline Ageratum conyzoides* & 0,11 & 3,97 & 0,058 & 4,84 \\
\hline Agave americana & 0,09 & 3,17 & 0,067 & 5,59 \\
\hline Andropogon sp.* & 0,13 & 4,76 & 0,031 & 2,58 \\
\hline Melinis minutiflora* & 0,09 & 3,17 & 0,036 & 3,01 \\
\hline Cnidoscolus urens* & 0,11 & 3,97 & 0,022 & 1,70 \\
\hline Vernonia scorpioides* & 0,07 & 2,38 & 0,028 & 2,35 \\
\hline Waltheria americana* & 0,07 & 2,38 & 0,024 & 2,05 \\
\hline Croton lundianus $*$ & 0,04 & 1,59 & 0,024 & 1,98 \\
\hline Emilia sonchifolia* & 0,07 & 2,38 & 0,011 & 0,90 \\
\hline Commelina erecta & 0,02 & 0,79 & 0,022 & 1,87 \\
\hline Lantana sp. & 0,02 & 0,79 & 0,022 & 1,87 \\
\hline Paspalum plicatulum* & 0,04 & 1,59 & 0,009 & 0,77 \\
\hline Stylosanthes viscosa* & 0,02 & 0,79 & 0,010 & 0,84 \\
\hline Doryopteris lorentzii & 0,02 & 0,79 & 0,003 & 0,28 \\
\hline Phyllanthus tenellus & 0,02 & 0,79 & 0,001 & 0,09 \\
\hline Chamaesyce sp. & 0,02 & 0,79 & 0,001 & 0,09 \\
\hline Centrosema brasilianum & 0,02 & 0,79 & 0,001 & 0,07 \\
\hline Total & 2,78 & 100,00 & 1,20 & 100,00 \\
\hline \multicolumn{5}{|c|}{$\begin{array}{l}\text { Espécies consideradas daninhas invasoras, segundo Lorenzi (2000) } \\
\text { e Kissman \& Groth (1997). }\end{array}$} \\
\hline
\end{tabular}


$\left(\mathrm{F}_{2,42}=2,31 ; \mathrm{p}<0,11 ; \mathrm{R}=0,31\right)$. A relação obtida foi representada pela fórmula:

Riqueza nos interceptos $=2,74+0,055 \times$ área da ilha - $0,028 \times$ profundidade da ilha, que explicou somente $0,05 \%$ da variação encontrada.

A relação gráfica obtida (Figura 1), considerando os efeitos parciais de cada uma das variáveis relacionadas com a riqueza, sugere que ela esteve mais correlacionada com a área da ilha do que com a profundidade do solo. A relação de acréscimo de riqueza a partir do aumento de tamanho insular foi encontrada em outros estudos realizados em afloramentos rochosos por Oliveira et al. (2004), em Minas Gerais, e Conceição et al. (2007) e Conceição \& Pirani (2007), na Bahia. No trabalho de Oliveira et al. (2004), a relação profundidade do solo e riqueza de espécies em ilhas de vegetação também se mostrou linear e significativa; ilhas com solos mais profundos apresentaram maior riqueza quando comparadas às ilhas de solos rasos.

A inexistência de relação entre o tamanho e profundidade das ilhas e a riqueza sugere que algum fator pode estar inibindo a recuperação da diversidade desse ecossistema. Segundo Benites et al. (2003), afloramentos rochosos são pobres em nutrientes em decorrência do forte processo de lixiviação promovido pelo relevo inclinado e pela baixa espessura do solo. Essas características podem fazer com que a infestação por plantas daninhas - eficientes colonizadoras desses ambientes - represente um forte fator inibidor da recuperação. A facilidade de germinação em um determinado ambiente tende a retardar a sucessão natural, por conta da competição por recursos e pela formação de densos aglomerados, que sufocam a vegetação nativa (Ziller, 2001). O efeito daninho dessas invasoras está refletido no forte papel inibidor do recrutamento de novas espécies e, consequentemente, de processos sucessionais (Connell \& Slatyer, 1977).

Novos estudos devem ser realizados para definir quais plantas, daninhas ou não, estão afetando o processo sucessional. Esse conhecimento poderia favorecer o desenvolvimento de protocolos que acelerem a recuperação da vegetação de inselbergs sob forte influência antrópica.
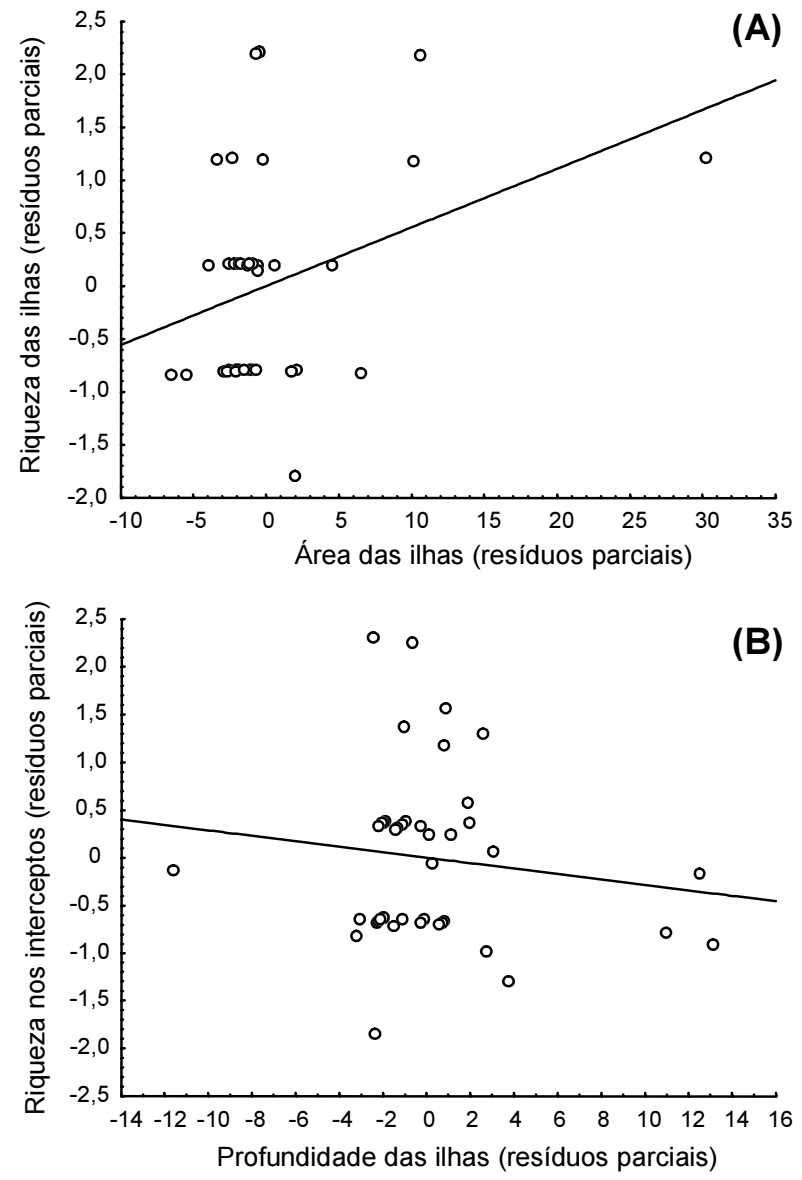

Figura 1 - Correlações dos efeitos parciais de cada uma das variáveis consideradas na regressão múltipla usada para explicar a riqueza nos interceptos, sendo: (A) correlação dos efeitos parciais da riqueza nos interceptos com a área das ilhas; e (B) correlação dos efeitos parciais da riqueza nos interceptos com a profundidade do solo das ilhas de vegetação do afloramento rochoso da Fazenda Estância Vale do Moxuara, Cariacica-ES, ano de 2009.

\section{AGRADECIMENTOS}

A Wilson Freitas Filho, que permitiu a realização deste trabalho em sua propriedade; a Marcelo Simonelli, pela ajuda na identificação das espécies; e a Jeohvá Junior, pela atenção e cooperação no trabalho de identificação realizado no herbário VIES da Universidade Federal do Espírito Santo (UFES).

\section{LITERATURA CITADA}

ANGIOSPERM PHYLOGENY GROUP. An update of the Angiosperm Phylogeny Group classification for the orders and families of flowering plants: APG II. Bot. J. Linn. Soc., v. 141, n. 4, p. 399-436, 2003.

Planta Daninha, Viçosa-MG, v. 29, n. 4, p. 745-750, 2011 
BENITES, V. M. et al. Solos e vegetação nos complexos rupestres de altitude da Mantiqueira e do Espinhaço. Flor. Amb., v. 10, n. 1, p. 76-85, 2003.

BROWER, J. E.; ZAR, J. H. Field and laboratory methods for general ecology. Dubuque: Wm. C. Brow Company Publishers, 1984. 226 p.

CAIAFA, A. N.; SILVA, A. F. Structural analysis of the vegetation on a highland granitic rock outcrop in Southeast Brazil. R. Bras. Bot., v. 30, n. 4, p. 657-664, 2007.

CONCEIÇÃO, A. A.; GIULIETTI, A. M. Composição florística e aspectos estruturais de campo rupestre em dois platôs do Morro do Pai Inácio, Chapada Diamantina, Bahia, Brasil. Hoehnea, v. 29, n. 1, p. 37-48, 2002

CONCEIÇÃO, A. A.; PIRANI, J. R. Diversidade em quatro áreas de campos rupestres na Chapada Diamantina, Bahia, Brasil: espécies distintas, mais riquezas similares

Rodriguésia, v. 58, n. 1, p. 193-206, 2007.

CONCEIÇÃO, A. A.; GIULIETTI, A. M.; MEIRELLES, S. T. Ilhas de vegetação em afloramentos rochosos de quartzitoarenito no Morro do Pai Inácio,Chapada Diamantina, Bahia, Brasil. Acta Bot. Bras., v. 21, n. 2, p. 335-347, 2007.

CONNELL J. H.; SLATYER, R. O. Mechanisms of succession in natural communities and their role in community stability and organization. Am. Nat., v. 111, n. 982, p. $1119-1144,1977$.

FERREIRA, E. A. et al. Estudos anatômicos de folhas de espécies de plantas daninhas: II - Bidens pilosa, Emilia sonchifolia, Ageratum conyzoides e Sonchus asper.

Planta Daninha, v. 20, n. 3, p. 327-335, 2002.

GRÖGER, A.; BARTHLOTT, W. Biogeography and diversity of the inselberg (Laja) vegetation of southern Venezuela. Biod. Letters, v. 3, n. 6, p. 165-179, 1996.

KISSMAN, K. G.; GROTH, D. Plantas infestantes e nocivas. São Paulo: BASF, 1997. 978 p.

LORENZI, H. Plantas daninhas do Brasil: terrestres, aquáticas, parasitas, tóxicas e medicinais. São Paulo, Plantarum, 2000. $608 \mathrm{p}$

MACEDO, J. F.; BRANDAO, M.; LARA, J. F. R. Plantas daninhas na pós-colheita de milho nas várzeas do rio São Francisco, em Minas Gerais. Planta Daninha, v. 21, n. 2, p. 239-248, 2003.
MODESTO JÚNIOR, M. S.; MASCARENHAS, R. E. B Levantamento da infestação de plantas daninhas associada a uma pastagem cultivada de baixa produtividade no Nordeste Paraense. Planta Daninha, v. 19, n. 1, p. 11-21, 2001.

OLIVEIRA, R. B.; GODOY, S. A. P. Composição florística dos afloramentos rochosos do Morro do Forno, Altinópolis, São Paulo. Biot. Neot., v. 7, n. 2, p. 37-47, 2007.

OLIVEIRA, T. D. et al. Estabelecimento de espécies vegetais em um inselberg granítico de Mata Atlântica. R. Est.. Biol., v. 26, n. 57 , p. $17-24,2004$

PEZZOPANE J. E. M. et al. Espacialização da temperatura do ar no estado do Espírito Santo. R. Bras. Agrometeorol., v. 12 , n. 1, p. $151-158,2004$

POREMBSKI, S. Tropical inselbergs: habitat types, adaptive strategies and diversity patterns. R. Bras. Bot., v. 30, n. 4, p. 579-586, 2007.

RADAMBRASIL. Levantamento dos recursos naturais: geologia, geomorfologia, pedologia, vegetação e uso potencial da terra. Rio de Janeiro, Ministério das Minas e Energia, 1983. p. SF. 23/24 - Rio de Janeiro/Vitória.

RIBEIRO, K. T; MEDINA, B. M. O. Estrutura, dinâmica e biogeografia das ilhas de vegetação sobre rocha do Planalto do Itatiaia, RJ. B. Parq. Nac. Itatiaia, v. 10, n. 1, p. 11-82, 2002.

RIBEIRO, K. T.; MEDINA, B. M. O.; SCARANO, F. R. Species composition and biogeographic relations of the rock outcrop flora on the high plateau of Itatiaia, SE-Brazil.

R. Bras. Bot., v. 30, n. 4, p. 623-639, 2007.

SCARANO, F. R. Structure, function and floristic relantioships of plants communities in stressful habitats marginal to Brazilian Atlantic rain forest. Ann. Bot., v. 90, n. 4 , p. $517-524,2002$

SMITH, A. R. et al. A classification for extant ferns. Taxon, v. 55, p. $705-731,2006$.

YAMASHITA, O. M. et al. Fatores ambientais sobre a germinação de Emilia sonchifolia. Planta Daninha, v. 27, n. 4, p. 673-681, 2009.

ZILLER, S. R. Plantas exóticas invasoras: A ameaça da contaminação biológica. R. Ci. Hoje, v. 30, n. 178, p. 77-79, 2001. 